\title{
The polymorphisms of HLA class I and II alleles were not associated with severe acute respiratory syndrome among recovered patients in Beijing: a case-control study
}

\author{
Dongmei $\mathrm{Li}^{1}$, Dongjing Liu ${ }^{2}$, Yi Zha ${ }^{1}$, Tao $\mathrm{Wu}^{2,3^{*}}$, Yan Qiu ${ }^{1 *}$ \\ ${ }^{1}$ Beijing Red Cross Blood Center, Beijing, 100088, China; \\ ${ }^{2}$ School of Public Health, Peking University, Beijing, 100191, China; \\ ${ }^{3}$ Key Laboratory of Reproductive Health, National Health Commission, Beijing, 100191, China
}

\begin{abstract}
Severe acute respiratory syndrome (SARS) is a viral respiratory illness caused by a novel coronavirus (SARS-CoV), which emerged as a pandemic in 2003. The mechanism of the immune reaction initiated by SARS-CoV still remains unclear. Here we aimed to describe the genetic patterns of high-resolution HLA-A, -B, -C, -DRB1, and -DQB1, loci in recovered SARS patients from Beijing and examine the association between HLA genes and susceptibility or resistance to SARS. A total of 70 recovered Chinese Han SARS patients were recruited to donate convalescent plasma in 2003. HLA high-resolution typing was carried out using sequence based typing (SBT). Allele frequencies were calculated by direct counting, and were compared with the frequencies of HLA alleles of donors recruited by the China Marrow Donor Program between 2002 and 2015 using Fisher's exact test. Significance of association was defined according to the Bonferroni method for multiple comparisons. We observed 20, 35, 21, 25, and 17 alleles respectively at HLA-A, -B, -C, -DRB1, and -DQB1 loci among the 70 recovered patients. We identified 12 alleles (HLA-A*02:10, -A*02:93, -A*03:02, -B*08:01, -B*15:152, -B*37:01, -DRB1*10:01, -DRB1*11:03, -DRB1*14:10, -DRB1*14:12, -DRB1*15:02, and -DQB1*05:10) showing a nominal association with SARS $(P<0.05)$, but none remained significant after Bonferroni correction. The study suggests that high-resolution HLA alleles are unlikely to contribute significantly to the susceptibility or resistance to SARS-CoV infection in the northern Chinese population.
\end{abstract}

Keywords: HLA, polymorphism, severe acute respiratory syndrome

\section{INTRODUCTION}

In November 2002, severe acute respiratory syndrome (SARS) broke out in Guangdong China, and rapidly spread to 29 countries/regions, resulting in 8,096 infections in 26 countries/regions, including 774 deaths according to the World Health Or- ganization (http://www.whoint/csr/sars/country/ table2004_04_21/en/). Beijing was one of the major epidemic areas in China and had the most severely affected population. Sixteen years later, although the immunological mechanism for SARS is still unclear, it is widely accepted that infectious diseases arise from a complex interaction between pathogens, environmen-

\footnotetext{
*Correspondence to: Tao Wu, Department of Epidemiology \& Biostatistics, School of Public Health, Peking University, 38 Xueyuan Road, Haidian District, Beijing, 100191, China. E-mail: twu@ @jmu.edu.cn; Yan Qiu, Beijing Red Cross Blood Center, 37 Bei San Huan Zhong Road, Haidian District, Beijing, 100088, China. E-mail: 13681201834@163.com.

Conflict of interests: The authors have declared no conflict of interests.
} 
tal factors and the host's genes of which the human leukocyte antigen (HLA) deserves careful attention. Several research groups have studied the association between HLA gene polymorphism and SARS, however inconsistent results have been reported ${ }^{[1-5]}$. One study showed a strong association of HLA class I allele B*0703 (OR: 4.08; 95\%CI, 2.03-8.18; $P=0.00072$ ) and class II allele DRBI*0301 (OR: 0.06; 95\% CI, 0.01$0.47 ; P=0.00008$ ) with the development of SARS in 90 Chinese patients ${ }^{[1]}$. Keicho et al. also suggested that a predominant allele, HLA-DRB $1 * 12$, in the Vietnamese population had a frequency of $46.6 \%$ in 88 SARS patients compared with a frequency of $28.7 \%$ in 101 healthy individuals who had contact with SARS $(P=0.0032)$ and $27.0 \%$ in 50 individuals without contact $(P=0.0053)^{[2]}$. In a Taiwanese group, HLA-C*0801 and $-C^{*} 1502$ showed to be associated with the SARS-CoV infection ${ }^{[3]}$. However, a cohort of 95 SARS patients suggested a lack of association of HLA-A, -B, and -DRB1 with the susceptibility or severity of SARS in Guangdong, China ${ }^{[4]}$. Our group previously performed HLA low-resolution typing assays for 148 recovered Chinese Han SARS patients by using PCR-SSP at the end of the SARS epidemic period. While Fisher's exact tests revealed four alleles (HLA-A*26, -DRB1*04, -DRB1*09, and -DRB1*16), showing nominal significance of association with the SARS infection $(P<0.05)$, none of these associations remained significant after correction for multiple comparisons ${ }^{[5]}$. Despite the above studies reporting possible SARS-associated HLA types in East Asian populations, there is currently a lack of relevant data on patients from Chinese Han population concerning HLA alleles such as HLA-DQBl in SARS patients.

In this study, we performed the high resolution genotyping of HLA class I (A, B, and C locus) and class II (DRB1 and $\mathrm{BQB} 1$ locus) genes using sequence based typing (SBT) among SARS recovered patients ${ }^{[4]}$, to investigate whether different HLA alleles profiles contribute to the risk of SARS infection among the Chinese Han population.

\section{MATERIALS AND METHODS}

\section{Subjects}

During the SARS epidemic, many recovering SARS patients donated plasma to treat other patients. This study involved the collection of whole blood samples from 70 such recovering patients according to the WHO definition of SARS and had been hospitalized in multiple designated SARS hospitals in Beijing between April and July 2003. All the participants were recruited and promoted by the Beijing Red Cross
Blood Center (BRCBC) to donate apheresis plasma in 2003 , for the purpose of preparing fresh frozen plasma with a potential SARS neutralizing antibody to treat SARS patients. All donors were screened according to the national standard GB18467-2001: Whole blood and component donor selection requirements, which included an informed consent sheet and a predonation questionnaire. The questions contained personal information, age, gender, place of residence and ethnic group etc. These 70 patients were all from Chinese Han population and were genetically unrelated, and met the physical examination criteria: negative screening results of HIV-Ab, HCV-Ab, HBsAg, syphilis, and alanine transaminase (ALT). Participants were then screened for the SARS antibody with SARS coronavirus (CoV)-Ab ELISA Kits ${ }^{[6]}$. Only those with positive SARS antibody were approved to donate plasma. This project was performed with the approval of the BRCBC Ethics Committee. Informed consent obtained indicated that the bio-sample would only be used for the purpose of SARS treatment and research.

\section{Sample collection and sample treatment}

The whole blood samples were collected in a vacuum container exposed to EDTA and transferred to the lab at $4{ }^{\circ} \mathrm{C}$. The plasma was separated for the antibody test. The buffy coats were lysed and genomic DNA was isolated using proteinase $\mathrm{K}$ treatment and the QIAGEN QIAamp DNA Blood Mini Kit (QIAGEN Inc., Valencia, CA, USA) according to the manufacturer's instructions. The DNA samples were divided and stored at $-80^{\circ} \mathrm{C}$ for future study.

\section{HLA high resolution typing}

The 70 stored genomic DNA samples were thawed. HLA high resolution typing was performed by SBT method with an Allele SEQR HLA Typing Kit (Celera Corporation, Alameda, CA, USA) according to the manufacturer's instructions.

\section{Controls}

The published frequencies of HLA-A, -B, -C, -DRB1, and -DQB1 alleles in unrelated Chinese bone marrow donors were used as control to compare the frequencies of HLA alleles found in the SARS group. These donors were recruited by the China Marrow Donor Program between 2002 and 2015. The sample size was 840,235 for HLA-A, HLA-B, HLA-DRB1, and 564,856 for HLA-C and HLA-DQB1. According to the donors' information, this donor group was predominantly Han Chinese (94.7\%) with an age ranged from 18 to 45 years. Since the majority of the patients in Medical Centers in Beijing come from north China 
and all the patients in the current study were Han Chinese, the control population was deemed reliable for comparison. HLA gene typing was carried out using SBT, and allele frequencies were estimated by direct counting. Hardy-Weinberg equilibrium testing yielded no significant deviations.

\section{Statistical analysis}

We directly counted HLA-A, -B, -C, -DRB1, and -DQB 1 alleles among the 70 subjects and calculated the allele frequencies as counts divided by $2 \times$ effective sample size. Odds ratios (OR) and the respective $95 \%$ confidence intervals(CI) were obtained from 2-by-2 table analysis. Two-tailed $P$ values were obtained using Fisher's exact test, by comparing the allele frequencies found in our group of patients with SARS versus the allele frequencies in the control population. To minimize type I error, we set the significance threshold as 0.05 divided by the number of alleles at every locus, according to Bonferroni correction ${ }^{[7]}$. The $P$ value thresholds for HLA-A, -B, -C, -DRB1, and -DQB1 loci were 0.05/20, 0.05/35, $0.05 / 21,0.05 / 25,0.05 / 17$, respectively. Statistical analyses were done on the open source environment $\mathrm{R}$ platform (Version 3.1.0).

\section{RESULTS}

In this patient cohort, there were 28 females and 42 males from 19 to 62 years old with an average of 33.2 years. HLA typing results were confirmed in 68 samples for HLA-A, -B, and -C alleles; 58 samples for HLA-DRBI; and 67 samples for HLA-DQB1. The reasons for HLA typing failure included inadequate DNA, PCR amplification failure, unable to read the SBT data, inconclusive SBT genotyping results, etc.

The allelic frequencies of HLA-A, -B, -C, -DRB1, and $-\mathrm{DQB} 1$ in high resolution (four-digit) were listed in Table 1-5, together with the frequencies of respective alleles in the control group, who were recruited by the China Marrow Donor Program between 2002 and 2015. 20,35, 21, 25, and 17 alleles were observed respectively at HLA-A, -B, -C, -DRB1, and -DQB1 loci. As shown in Table 1-5, there was no significant difference between the SARS patient group and control group for HLA-C allele frequency. However, for HLA-A, -B, -DRB1, and -DQB1 alleles, we saw a nominal significant difference. The frequencies were as follows: HLA-A*03:02 (OR: 10.16, 95\%CI: 2.0730.36, $P=0.0036$ ); HLA-A*02:10 (OR: 6.37, 95\%CI: 1.30-19.03, $P=0.0127$ ); HLA-A*02:93 (OR: 148.26, 95\% CI: 3.68-868.58; $P=0.0069)$; HLA-B*37:01 (OR: $2.69,95 \%$ CI: $0.86-6.44, P=0.0430$ ); HLAB*08:01 (OR: 4.99, 95\%CI: 1.80-11.18, $P=0.0017$ ); HLA-B*15:152(OR: 946.67, 95\%CI: 22.378,192.00, $P=0.0011$ ); HLA-DRB1*10:01 (OR: 3.39, 95\%CI: $1.22-7.61, P=0.0107)$; HLA-DRB1 $* 11: 03$ (OR: 23.15, 95\%CI: 0.58-132.14; $P=0.0427$ ); HLA-DRB ${ }^{*} 15: 02$ (OR: $2.28,95 \%$ CI: $0.96-4.66$, $P=0.0305)$; HLA-DRB1 $* 14: 10$ (OR: $33.97,95 \% \mathrm{CI}$ : $0.85-194.28, P=0.0293$ ); HLA-DRB $1 * 14: 12$ (OR: $21.8,95 \%$ CI: $0.55-124.60, P=0.0453)$; HLADQB1*05:10 (OR: 28.78, 95\%CI: 0.72-163.82, $P=0.0345)$. These results showed that the frequen-

Table 1 The results of association test for HLA-A alleles

\begin{tabular}{|c|c|c|c|c|c|c|}
\hline HLA alleles & & Number of alleles & $\begin{array}{c}\text { Frequency in case } \\
\text { sample }(\%)\end{array}$ & $\begin{array}{c}\text { Frequency in control } \\
\text { sample }(\%)\end{array}$ & $\mathrm{OR}(95 \% \mathrm{CI})$ & $P^{\mathrm{a}}$ \\
\hline \multirow{20}{*}{$\begin{array}{l}\text { HLA-A } \\
\text { Case } 2 n=136, \\
\text { Control } 2 n=1,680,470, \\
P=0.0025\end{array}$} & 01:01 & 7 & 5.1 & 3.6 & $1.46(0.58,3.10)$ & 0.3474 \\
\hline & $01: 69$ & 1 & 0.7 & 0.0 & - & - \\
\hline & 02:01 & 16 & 11.8 & 12.0 & $0.98(0.54,1.65)$ & 1.0000 \\
\hline & 02:03 & 4 & 2.9 & 3.5 & $0.84(0.23,2.20)$ & 1.0000 \\
\hline & 02:06 & 9 & 6.6 & 5.2 & $1.29(0.58,2.53)$ & 0.4369 \\
\hline & 02:07 & 10 & 7.4 & 8.4 & $0.86(0.40,1.64)$ & 0.7586 \\
\hline & $02: 10$ & 3 & 2.2 & 0.4 & $6.37(1.30,19.03)$ & 0.0127 \\
\hline & 02:93 & 1 & 0.7 & 0.0 & $148.26(3.68,868.58)$ & 0.0069 \\
\hline & 03:01 & 6 & 4.4 & 3.0 & $1.51(0.54,3.38)$ & 0.3032 \\
\hline & 03:02 & 3 & 2.2 & 0.2 & $10.16(2.07,30.36)$ & 0.0036 \\
\hline & 11:01 & 23 & 16.9 & 20.9 & $0.77(0.47,1.21)$ & 0.2917 \\
\hline & 11:02 & 2 & 1.5 & 1.8 & $0.84(0.10,3.08)$ & 1.0000 \\
\hline & $24: 02$ & 28 & 20.6 & 15.5 & $1.41(0.90,2.15)$ & 0.1223 \\
\hline & $24: 20$ & 1 & 0.7 & 0.3 & $2.84(0.07,16.1)$ & 0.2984 \\
\hline & $26: 01$ & 1 & 0.7 & 2.8 & $0.25(0.01,1.44)$ & 0.1927 \\
\hline & 29:01 & 1 & 0.7 & 0.7 & $1.10(0.03,6.21)$ & 0.5999 \\
\hline & 30:01 & 4 & 2.9 & 5.9 & $0.48(0.13,1.26)$ & 0.1987 \\
\hline & $31: 01$ & 6 & 4.4 & 3.3 & $1.36(0.49,3.05)$ & 0.4615 \\
\hline & $32: 01$ & 1 & 0.7 & 1.3 & $0.55(0.01,3.11)$ & 1.0000 \\
\hline & $33: 03$ & 9 & 6.6 & 8.2 & $0.80(0.36,1.56)$ & 0.6382 \\
\hline
\end{tabular}

${ }^{\mathrm{a}}$ : 2-tailed value, by Fisher's exact test, alleles with nominal significance were marked in bold. 

syndrome among recovered patients in Beijing: A case-control study, 2019, 3(1)

Table 2 The results of association test for HLA-B alleles

\begin{tabular}{|c|c|c|c|c|c|c|}
\hline HLA alleles & & Number of alleles & $\begin{array}{c}\text { Frequency in case } \\
\text { sample }(\%)\end{array}$ & $\begin{array}{c}\text { Frequency in control } \\
\text { sample }(\%)\end{array}$ & $\mathrm{OR}(95 \% \mathrm{CI})$ & $P^{\mathrm{a}}$ \\
\hline \multirow{35}{*}{$\begin{array}{l}\text { HLA-B } \\
\text { Case } 2 n=136, \\
\text { Control } 2 n=1,680,470, \\
P=0.0014\end{array}$} & 07:02 & 2 & 1.5 & 2.1 & $0.69(0.08,2.54)$ & 1.0000 \\
\hline & 07:05 & 1 & 0.7 & 0.5 & $1.38(0.03,7.85)$ & 0.5160 \\
\hline & 08:01 & 6 & 4.4 & 0.9 & $4.99(1.80,11.18)$ & 0.0017 \\
\hline & 13:01 & 7 & 5.1 & 5.0 & $1.02(0.40,2.17)$ & 0.8448 \\
\hline & 13:02 & 6 & 4.4 & 6.3 & $0.69(0.25,1.54)$ & 0.4789 \\
\hline & 15:01 & 11 & 8.1 & 4.8 & $1.76(0.85,3.25)$ & 0.1016 \\
\hline & 15:02 & 8 & 5.9 & 3.6 & $1.68(0.71,3.41)$ & 0.1594 \\
\hline & $15: 11$ & 4 & 2.9 & 1.8 & $1.62(0.43,4.24)$ & 0.3218 \\
\hline & $15: 13$ & 1 & 0.7 & 0.1 & $13.59(0.34,77.29)$ & 0.0715 \\
\hline & $15: 152$ & 1 & 0.7 & 0.0 & $946.67(22.37,8192)$ & 0.0011 \\
\hline & $15: 18$ & 2 & 1.5 & 1.4 & $1.08(0.13,3.98)$ & 0.7092 \\
\hline & $27: 04$ & 1 & 0.7 & 0.9 & $0.79(0.02,4.50)$ & 1.0000 \\
\hline & $27: 05$ & 1 & 0.7 & 0.7 & $1.02(0.03,5.77)$ & 0.6268 \\
\hline & $35: 01$ & 4 & 2.9 & 2.4 & $1.21(0.33,3.18)$ & 0.5773 \\
\hline & $35: 03$ & 2 & 1.5 & 1.1 & $1.32(0.16,4.87)$ & 0.6665 \\
\hline & $35: 08$ & 1 & 0.7 & 0.1 & $5.11(0.13,28.97)$ & 0.1788 \\
\hline & $37: 01$ & 5 & 3.7 & 1.4 & $2.69(0.86,6.44)$ & 0.0430 \\
\hline & 38:01 & 1 & 0.7 & 0.5 & $1.45(0.04,8.21)$ & 0.5002 \\
\hline & 38:02 & 5 & 3.7 & 2.6 & $1.43(0.46,3.41)$ & 0.4096 \\
\hline & 39:01 & 2 & 1.5 & 1.8 & $0.83(0.10,3.05)$ & 1.0000 \\
\hline & 39:05 & 1 & 0.7 & 0.2 & $4.25(0.11,24.08)$ & 0.2111 \\
\hline & 40:01 & 9 & 6.6 & 9.6 & $0.67(0.30,1.31)$ & 0.3062 \\
\hline & 40:02 & 2 & 1.5 & 1.9 & $0.78(0.09,2.89)$ & 1.0000 \\
\hline & 40:06 & 6 & 4.4 & 3.2 & $1.39(0.50,3.10)$ & 0.4576 \\
\hline & 44:02 & 1 & 0.7 & 0.9 & $0.84(0.02,4.78)$ & 1.0000 \\
\hline & 44:03 & 3 & 2.2 & 2.7 & $0.80(0.16,2.39)$ & 1.0000 \\
\hline & $46: 01$ & 13 & 9.6 & 10.3 & $0.92(0.48,1.64)$ & 0.8881 \\
\hline & 48:01 & 3 & 2.2 & 2.5 & $0.89(0.18,2.67)$ & 1.0000 \\
\hline & $51: 01$ & 9 & 6.6 & 5.6 & $1.19(0.53,2.33)$ & 0.5749 \\
\hline & $51: 02$ & 2 & 1.5 & 1.1 & $1.40(0.17,5.16)$ & 0.6573 \\
\hline & $52: 01$ & 5 & 3.7 & 3.0 & $1.25(0.40,2.98)$ & 0.6072 \\
\hline & $54: 01$ & 4 & 2.9 & 3.1 & $0.94(0.25,2.47)$ & 1.0000 \\
\hline & $55: 02$ & 1 & 0.7 & 2.5 & $0.29(0.01,1.65)$ & 0.2700 \\
\hline & $57: 01$ & 1 & 0.7 & 1.2 & $0.63(0.02,3.56)$ & 1.0000 \\
\hline & $58: 01$ & 5 & 3.7 & 6.1 & $0.58(0.19,1.40)$ & 0.2847 \\
\hline
\end{tabular}

${ }^{\mathrm{a}}$ : 2-tailed value, by Fisher's exact test, alleles with nominal significance were marked in bold.

cies of SARS group were higher than those of control group with $P<0.05$. However, none of these associations remained significant after Bonferroni correction.

\section{DISCUSSION}

In this study, HLA allele high resolution typing was performed on samples from 70 SARS patients and their frequencies were compared with donors from the China Marrow Donor Program between 2002 and 2015. 3 alleles of HLA-A, 2 alleles of HLA-B, 5 alleles of HLA-DRB 1 and 1 allele of HLA-DQB1 showed nominal significance. Among these 12 nominal associations, HLA-A*03:02, -A*02:10, -B*37:01, -B*08:01, -DRB1*10:01, and -DRB1*15:02 may have possible associations with a susceptibility to SARS, while the other 6 alleles only presented once in our population and also had low frequencies in the control group, which makes it difficult to speculate on the relationship between HLA alleles and infectious diseases. None of these associations remained significant after Bonferroni correction for multiple comparisons.

The HLA system plays an essential role in the immune system and the host's response to viral infections. HLA gene products include antigenic peptides in response to $\mathrm{T}$ cells, which are involved in immune response initiation and foreign material cleaning by neutralizing antibodies, cytokines, and activated cytotoxic $\mathrm{T}$ cells. The host immune condition is also critical for the susceptibility to certain viral infections. Therefore, individual susceptibility or resistance to immune-mediated/controlled diseases may be influenced by the polymorphisms of alleles in the HLA system. HLA genes form part of a highly polymorphic 
The polymorphisms of HLA class I and II alleles were not associated with severe acute respiratory syndrome among recovered patients in Beijing: A case-control study, 2019, 3(1)

Table 3 The results of association test for HLA-C alleles

\begin{tabular}{|c|c|c|c|c|c|c|}
\hline HLA alleles & & Number of alleles & $\begin{array}{c}\text { Frequency in case } \\
\text { sample }(\%)\end{array}$ & $\begin{array}{c}\text { Frequency in contro } \\
\text { sample }(\%)\end{array}$ & $\mathrm{OR}(95 \% \mathrm{CI})$ & $P^{\mathrm{a}}$ \\
\hline \multirow{21}{*}{$\begin{array}{l}\text { HLA-C } \\
\text { Case } 2 n=136, \\
\text { Control } 2 n=1,129,712, \\
P=0.0024\end{array}$} & 01:02 & 23 & 16.9 & 15.9 & $1.08(0.66,1.70)$ & 0.7251 \\
\hline & 01:03 & 2 & 1.5 & 0.6 & $2.36(0.28,8.70)$ & 0.2108 \\
\hline & 02:02 & 1 & 0.7 & 0.7 & $1.07(0.03,6.04)$ & 0.6101 \\
\hline & 03:02 & 5 & 3.7 & 5.9 & $0.61(0.19,1.45)$ & 0.3616 \\
\hline & 03:03 & 12 & 8.8 & 6.9 & $1.30(0.65,2.35)$ & 0.3953 \\
\hline & 03:04 & 14 & 10.3 & 9.9 & $1.04(0.55,1.82)$ & 0.8856 \\
\hline & 04:01 & 6 & 4.4 & 5.8 & $0.75(0.27,1.69)$ & 0.7108 \\
\hline & 06:02 & 12 & 8.8 & 8.9 & $1.00(0.50,1.80)$ & 1.0000 \\
\hline & 07:02 & 19 & 14.0 & 15.2 & $0.91(0.53,1.48)$ & 0.8110 \\
\hline & 07:04 & 1 & 0.7 & 0.9 & $0.85(0.02,4.79)$ & 1.0000 \\
\hline & 07:06 & 1 & 0.7 & 0.8 & $0.92(0.02,5.21)$ & 1.0000 \\
\hline & 08:01 & 15 & 11.0 & 8.5 & $1.33(0.72,2.28)$ & 0.2827 \\
\hline & 08:03 & 2 & 1.5 & 0.8 & $1.91(0.23,7.04)$ & 0.2842 \\
\hline & $08: 22$ & 2 & 1.5 & 0.9 & $1.70(0.20,6.26)$ & 0.3319 \\
\hline & 12:02 & 6 & 4.4 & 3.4 & $1.31(0.47,2.93)$ & 0.4724 \\
\hline & 12:03 & 1 & 0.7 & 1.9 & $0.38(0.01,2.13)$ & 0.5282 \\
\hline & 14:02 & 7 & 5.1 & 4.3 & $1.21(0.48,2.57)$ & 0.5263 \\
\hline & $14: 03$ & 2 & 1.5 & 1.0 & $1.45(0.17,5.36)$ & 0.4027 \\
\hline & $15: 02$ & 3 & 2.2 & 3.3 & $0.65(0.13,1.95)$ & 0.6338 \\
\hline & $15: 05$ & 1 & 0.7 & 0.7 & $1.05(0.03,5.95)$ & 0.6156 \\
\hline & $16: 04$ & 1 & 0.7 & 0.1 & $9.45(0.24,53.63)$ & 0.1012 \\
\hline
\end{tabular}

a: 2-tailed value, by Fisher's exact test, alleles with nominal significance were marked in bold.

Table 4 The results of association test for HLA-DRB1 alleles

\begin{tabular}{|c|c|c|c|c|c|c|}
\hline HLA alleles & & Number of alleles & $\begin{array}{c}\text { Frequency in case } \\
\text { sample }(\%)\end{array}$ & $\begin{array}{c}\text { Frequency in control } \\
\text { sample }(\%)\end{array}$ & OR(95\%CI) & $P^{\mathrm{a}}$ \\
\hline \multirow{25}{*}{$\begin{array}{l}\text { HLA-DRB } 1 \\
\text { Case } 2 n=116, \\
\text { Control } 2 n=1,680,470, \\
P=0.0020\end{array}$} & 01:01 & 2 & 1.7 & 2.1 & $0.83(0.10,3.08)$ & 1.0000 \\
\hline & 03:01 & 4 & 3.4 & 5.1 & $0.66(0.18,1.74)$ & 0.5301 \\
\hline & 04:01 & 1 & 0.9 & 1.0 & $0.86(0.02,4.91)$ & 1.0000 \\
\hline & 04:02 & 1 & 0.9 & 0.2 & $4.95(0.12,28.15)$ & 0.1844 \\
\hline & 04:03 & 1 & 0.9 & 1.6 & $0.55(0.01,3.12)$ & 1.0000 \\
\hline & 04:05 & 6 & 5.2 & 4.8 & $1.08(0.39,2.43)$ & 0.8263 \\
\hline & 04:06 & 1 & 0.9 & 2.6 & $0.33(0.01,1.88)$ & 0.3773 \\
\hline & 07:01 & 7 & 6.0 & 9.6 & $0.60(0.24,1.29)$ & 0.2672 \\
\hline & 08:03 & 7 & 6.0 & 6.3 & $0.96(0.38,2.04)$ & 1.0000 \\
\hline & 09:01 & 24 & 20.7 & 14.7 & $1.51(0.92,2.39)$ & 0.0869 \\
\hline & 10:01 & 6 & 5.2 & 1.6 & $3.39(1.22,7.61)$ & 0.0107 \\
\hline & 11:01 & 3 & 2.6 & 5.6 & $0.44(0.09,1.33)$ & 0.2221 \\
\hline & 11:03 & 1 & 0.9 & 0.0 & $23.15(0.58,132.14)$ & 0.0427 \\
\hline & 12:01 & 3 & 2.6 & 2.4 & $1.07(0.22,3.20)$ & 0.7614 \\
\hline & $12: 02$ & 8 & 6.9 & 8.7 & $0.78(0.33,1.58)$ & 0.6209 \\
\hline & $12: 10$ & 1 & 0.9 & 0.2 & $3.61(0.09,20.55)$ & 0.2433 \\
\hline & $13: 02$ & 3 & 2.6 & 3.3 & $0.78(0.16,2.33)$ & 1.0000 \\
\hline & $13: 12$ & 3 & 2.6 & 0.7 & $3.54(0.72,10.60)$ & 0.0565 \\
\hline & 14:05 & 2 & 1.7 & 2.2 & $0.78(0.09,2.89)$ & 1.0000 \\
\hline & $14: 10$ & 1 & 0.9 & 0.0 & $33.97(0.85,194.28)$ & 0.0293 \\
\hline & $14: 12$ & 1 & 0.9 & 0.0 & $21.8(0.55,124.60)$ & 0.0453 \\
\hline & $14: 54$ & 1 & 0.9 & 2.4 & $0.35(0.01,1.99)$ & 0.5354 \\
\hline & 15:01 & 15 & 12.9 & 11.6 & $1.13(0.61,1.96)$ & 0.6626 \\
\hline & $15: 02$ & 8 & 6.9 & 3.1 & $2.28(0.96,4.66)$ & 0.0305 \\
\hline & 16:02 & 6 & 5.2 & 3.1 & $1.71(0.62,3.85)$ & 0.1763 \\
\hline
\end{tabular}

${ }^{\mathrm{a}}$ : 2-tailed value, by Fisher's exact test, alleles with nominal significance were marked in bold.

system and many HLA alleles have been shown to be associated with susceptibility or resistance to a wide range of infections, including HIV type $1^{[8]}$, HTLV type $1^{[9]}, \mathrm{HCV}^{[10]}$ and malaria ${ }^{[11]}$.
In this context, many researchers have investigated the association between different HLA class I and II alleles and SARS after its outbreak in 2003. However, until now there has been no consensus regarding such 

syndrome among recovered patients in Beijing: A case-control study, 2019, 3(1)

Table 5 The results of association test for HLA-DQB1 alleles

\begin{tabular}{|c|c|c|c|c|c|c|}
\hline HLA alleles & & Number of alleles & $\begin{array}{c}\text { Frequency in case } \\
\text { sample }(\%)\end{array}$ & $\begin{array}{c}\text { Frequency in control } \\
\text { sample }(\%)\end{array}$ & $\mathrm{OR}(95 \% \mathrm{CI})$ & $P^{\mathrm{a}}$ \\
\hline \multirow{17}{*}{$\begin{array}{l}\text { HLA-DQB } 1 \\
\text { Case } 2 n=134, \\
\text { Control } 2 n=1,068,286, \\
P=0.0030\end{array}$} & 02:01 & 7 & 5.2 & 4.9 & $1.06(0.42,2.25)$ & 0.8406 \\
\hline & 02:02 & 8 & 6.0 & 7.6 & $0.77(0.33,1.57)$ & 0.6236 \\
\hline & 03:01 & 27 & 20.1 & 21.1 & $0.94(0.60,1.45)$ & 0.9156 \\
\hline & 03:02 & 3 & 2.2 & 5.7 & $0.38(0.08,1.12)$ & 0.0924 \\
\hline & 03:03 & 27 & 20.1 & 15.9 & $1.34(0.84,2.05)$ & 0.1924 \\
\hline & 03:05 & 1 & 0.7 & 0.1 & $8.03(0.20,45.6)$ & 0.1179 \\
\hline & 04:01 & 7 & 5.2 & 4.5 & $1.17(0.46,2.48)$ & 0.6735 \\
\hline & 04:02 & 1 & 0.7 & 1.3 & $0.59(0.01,3.35)$ & 1.0000 \\
\hline & 05:01 & 10 & 7.5 & 4.6 & $1.66(0.78,3.15)$ & 0.1437 \\
\hline & 05:02 & 6 & 4.5 & 7.3 & $0.60(0.21,1.33)$ & 0.2466 \\
\hline & 05:03 & 3 & 2.2 & 4.2 & $0.52(0.11,1.57)$ & 0.3840 \\
\hline & 05:10 & 1 & 0.7 & 0.0 & $28.78(0.72,163.82)$ & 0.0345 \\
\hline & 06:01 & 15 & 11.2 & 10.2 & $1.11(0.60,1.90)$ & 0.6691 \\
\hline & 06:02 & 14 & 10.4 & 7.6 & $1.41(0.75,2.47)$ & 0.2506 \\
\hline & $06: 03$ & 1 & 0.7 & 1.5 & $0.50(0.01,2.85)$ & 1.0000 \\
\hline & 06:04 & 2 & 1.5 & 1.4 & $1.04(0.12,3.83)$ & 0.7189 \\
\hline & 06:09 & 1 & 0.7 & 1.7 & $0.44(0.01,2.48)$ & 0.7318 \\
\hline
\end{tabular}

a: 2-tailed value, by Fisher's exact test, alleles with nominal significance were marked in bold.

reports from Taiwan ${ }^{[3,12]}$, Hong Kong ${ }^{[1]}$, and Guangdong ${ }^{[4]}$. Similar to our findings, a report of a SARS cohort comprised of 95 recovered SARS patients from Guangzhou in Southern China demonstrated no significant association ${ }^{[4]}$. However, the nominal significant HLA alleles found in our study and the Guangzhou study had no overlap at all. A previous study ${ }^{[12]}$ performing HLA class I and II allele typing was conducted on 37 probable SARS Taiwan patients. The results indicated that $\mathrm{HLA}-\mathrm{B} * 46: 01(\mathrm{OR}=2.08, P=$ $0.04, P c=$ n.s. $)$ and HLA-B $* 54: 01(\mathrm{OR}=5.44, P=$ $0.02, P c=$ n.s.) could be the most likely factors favoring SARS-CoV infection, but only HLA-B*46:01 was significantly associated with severe SARS cases $(P=$ 0.0008 or $P c=0.0279$ ). Another study in the Asian population was performed on 44 Vietnamese SARS patients, which reported HLA-DRB1*12:02 to be a new candidate allele involved in SARS development ${ }^{[2]}$. In our study, the frequencies of HLA-DRB1*12:02 alleles showed no significant difference between the SARS cases and control group. Another study reported that among 90 SARS patients in Hong Kong, those with HLA-B*07:03 were susceptible to the development of SARS while those with HLA-DRB $1 * 03: 01$ genotypes were resistant to SARS-CoV infection ${ }^{[1]}$. A later study conducted on 130 cases in Taiwan also suggested HLA-C $* 15: 02$ and -DRBI*03:01 alleles conferred factors for resistance against SARS infection $(P=0.04)^{[13]}$. In this study, however, HLA-B*07:03 did not occur in the enrolled SARS population. Although the frequencies of HLA-C*15:02 and -DRB1*03:01 were lower in SARS patients than in the control group respectively, there was no statistical significance between the two groups after applying Bonferroni correction. Popula- tions from mainland China, Hong Kong and Taiwan largely share common origins with the Vietnamese, whose DNA has been influenced by residents from southern China since the Bronze Age ${ }^{[14]}$. However, SARS-associated HLA alleles reported in any one of these populations could not be verified in the other populations. These inconsistencies among studies may have resulted from the limited sample size, different typing method, and various choices of control, HLA heterogeneity, or diversity in SARS-CoV strains. For example, the enrolled patients in our study were composed of recovered SARS patients, while the allele frequencies from the control group were from unrelated Chinese bone marrow donors. Lin et al. ${ }^{[3]}$ investigated a population of 37 cases of probable SARS, plus 28 patients with fevers excluded later as probable SARS, and 101 non-infected health care workers as control. $\mathrm{Ng}$ et al. ${ }^{[1]}$ described the association of some HLA alleles with a susceptibility or resistance to the SARS infection in a population of 90 Chinese patients when compared with unrelated Hong Kong Chinese bone marrow donors. The study performed in Guangdong also enrolled 95 cases of recovered SARS patients and compared their HLA allele frequencies with those of 403 unrelated healthy volunteers from the same geographical area. However, the severity of the SARS infections and the different nature of the control group may have led to the identification of distinct alleles.

This study employed a large sample of Chinese bone marrow donors as the control group, which outnumbered the sample size of the case group dramatically. However, we believe this imbalanced case : control ratio did not skew our analysis. The predomi- 
nant Han ancestry of the control cohort rendered a reliable comparison with the case group, since Chinese Han in north China is a relatively homogenous population, as suggested by both population history and modern genetic studies.

The mechanism behind HLA type and SARS pathogenesis still remains elusive and warrants further investigation. As cell surface proteins, HLA participate in establishing an antigen specific T-cell repertoire and subsequently activate such $\mathrm{T}$ cells during the initiation of immune responses. Class I (HLA-A, -B, and $-\mathrm{C}$ ) are responsible for $\mathrm{CD} 8 \mathrm{-T}$ cell responses and II (HLA-DR and -DQ) for CD4-T cell responses. Although as yet, we do not know the molecular details of the immune response to SARS-CoV infection clearly, it is believed that the innate inflammatory immune response, rather than the specific arm of the immune response function, is the more important determinant ${ }^{[15]}$. Moreover, intrinsic factors such as age and associated underlying illness were also suggested to be of importance ${ }^{[16]}$. From this perspective, the non-significant results of the present data are consistent with these current views, and there remains little or no available evidence to understand the role of naturally acquired immune responses in the re-infection of SARS-CoV. Therefore further research into HLA and SARS-CoV infection, development, and clearance needs to be conducted for effective vaccination strategy.

In summary, this study did not find any significant association between polymorphisms of HLA and the risk of SARS infection in SARS recovered patients from Beijing Medical Centers when using the Bonferroni correction method. So, further studies with a relatively larger sample size need to be conducted to confirm the finding of the current study.

\section{References}

[1] $\mathrm{Ng} \mathrm{MH,} \mathrm{Lau} \mathrm{KM,} \mathrm{Li} \mathrm{L,} \mathrm{et} \mathrm{al.} \mathrm{Association} \mathrm{of} \mathrm{hu-}$ man-leukocyte-antigen class I (B*0703) and class II (DRB1*0301) genotypes with susceptibility and resistance to the development of severe acute respiratory syndrome. J Infect Dis, 2004, 190(3): 515-8.

[2] Keicho N, Itoyama S, Kashiwase K, et al. Association of human leukocyte antigen class II alleles with severe acute respiratorysyndrome in the Vietnamese population. Hum Immunol, 2009, 70(7):527-31.
[3] Chen YM, Liang SY, Shih YP, et al. Epidemiological and genetic correlates of severe acute respiratory syndrome coronavirusinfection in the hospital with the highest nosocomial infection rate in Taiwan in 2003. J Clin Microbiol, 2006, 44(2):359-65.

[4] Xiong P, Zeng X, Song MS, et al. Lack of association between HLA-A, -B and-DRB1 alleles and the development of SARS: acohort of 95 SARS-recovered individuals in a population of Guangdong, southern China. Int $J$ Immunogenet, 2008, 35(1):69-74.

[5] Liu DJ, Qiu Y, Zha Y, et al. Association of HLA class I and class II genes with severe acute respiratory syndrome in the northern Chinese population. Asia-Pacific Journal of Blood Types and Genes, 2018, 2(2):91-6.

[6] Gao GJ, Qiu Y, Zhang P, et al. Investigation on SARS in Beijing volunteer blood donors. Chin J Blood Transfusion, 2003, 16(4):223-6.

[7] Bland JM, Altman DG. Multiple significance tests: the Bonferroni method. BMJ, 1995, 310:170.

[8] Carrington M, O’Brien SJ. The influence of HLA genotype on AIDS. Annu Rev Med, 2003, 54:535-51.

[9] Jeffery KJ, Siddiqui AA, Bunce M, et al. The influence of HLA class I alleles and heterozygosity on the outcome of human T cell lymphotropic virus type I infection. J Immunol, 2000, 165:7278-84.

[10] Khakoo SI, Thio CL, Martin MP, et al. HLA and NK cell inhibitory receptor genes in resolving hepatitis $\mathrm{C}$ virus infection. Science, 2004, 305:872-4.

[11] Martin MP, Carrington M. Immunogenetics of viral infections. Curr Opin Immunol, 2005, 17:510-6.

[12] Lin M, Tseng HK, Trejaut JA, et al. Association of HLA class I with severe acute respiratory syndrome coronavirus infection. BMC Med Genet, 2003, 4:9.

[13] Wang SF, Chen KH, Chen M, et al. Human-leukocyte antigen class I Cw 1502 and class II DR 0301 genotypes are associated with resistance to severe acute respiratory syndrome (SARS) infection. Viral Immunol, 2011, 24:421-6.

[14] Hoa BK, Hang NT, Kashiwase K, et al. HLA-A, -B, -C, -DRB1 and -DQB1 alleles and haplotypes in the Kinh population in Vietnam. Tissue Antigens, 2008, 71:127-34.

[15] Reghunathan R, Jayapal M, Hsu LY, et al. Expression profile of immune response genes in patients with severe acute respiratory syndrome. BMC Immunol, 2005, 6:2.

[16] Peiris JSM, Guan Y, Yuen KY. Severe acute respiratory syndrome. Nat Med, 2004, 10(12 Suppl):S88-97.

(Received 28 December 2018, Revised 12 February 2019, Accepted 11 March 2019) 


\title{
Antibody
} 这迪溥猪

\section{JiangSu LIBO Medicine Biotechnology Co.,Ltd.}

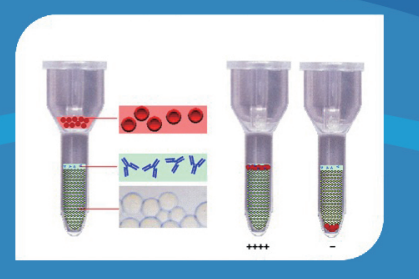

\author{
Microcolumn Gel Technology
}

\section{Professional Supplier of \\ Blood Diagnostic Reagents}
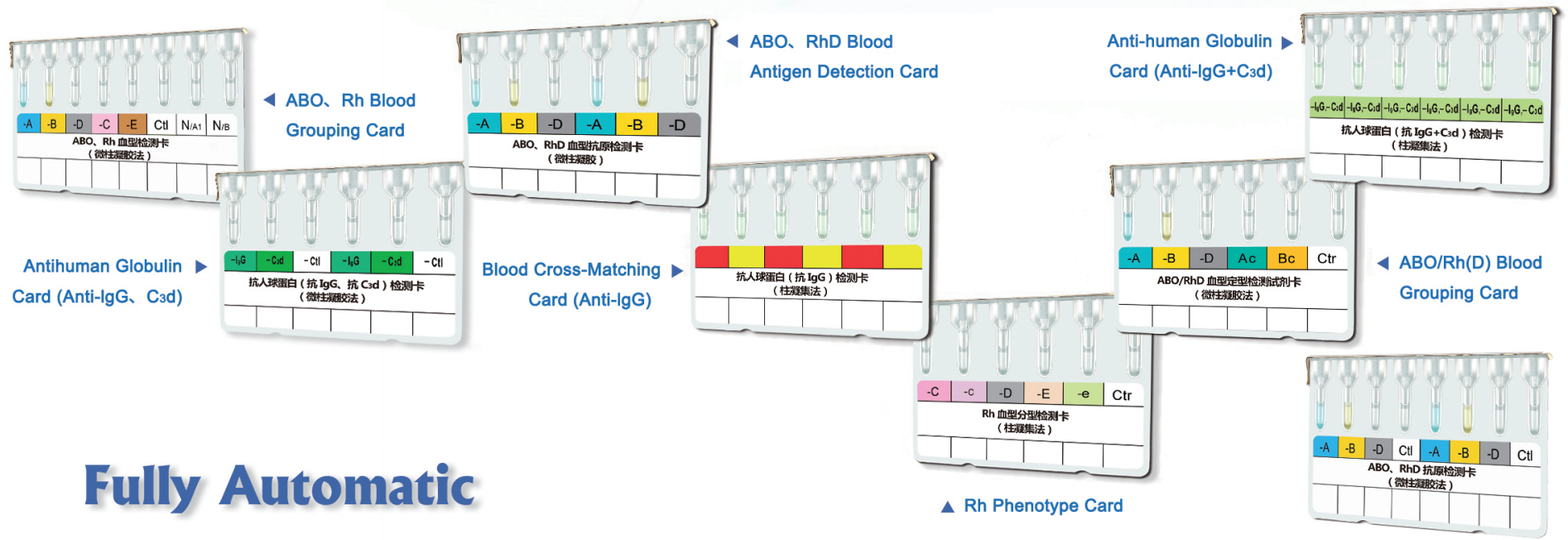

\section{Blood Grouping Analysis System}

A ABO, RhD Blood Detection Card

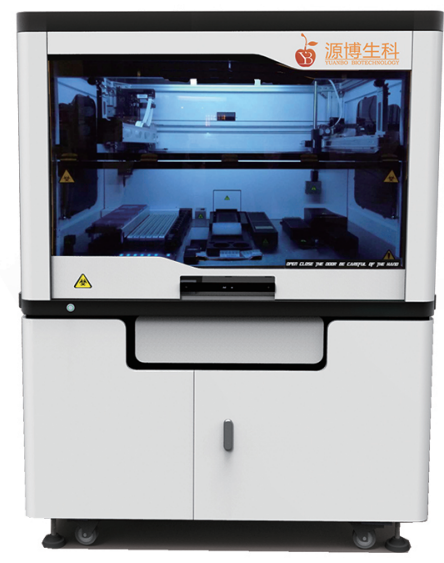

- Microcolumn gel immunoassay for

$\mathrm{ABO} / \mathrm{Rh}(\mathrm{D})$ blood grouping; Cross matching;

Antibody screening

- High throughout w/DUAL-centrifuge design

- Accuracy of sampling $\leq 2 \%$;

Repeatability of sampling CV $\leq 1.5 \%$

- Auto result acquisition and interpretation by CCD imaging system

\section{Antibod"} 安画溥猜

JiangSu LIBO Medicine Biotechnology Co.,Ltd.

Address: No 78 West Dongsheng Road, Jiangyin, Jiangsu214400, P.R. China

Web Site: www.libiotech.com

Sales Tel: +86-510-86990618、+86-510-86990633

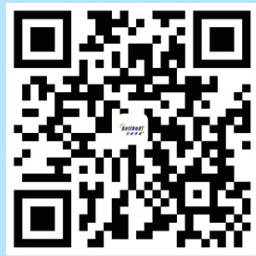

Tech Tel: +86-510-86990608、+86-510-86990655 